\title{
GROUPS WITH ONLY RESOLVABLE GROUP TOPOLOGIES
}

\author{
W. W. COMFORT AND JAN VAN MILL
}

(Communicated by Franklin D. Tall)

\begin{abstract}
Adapting terminology suggested by work of E. Hewitt [Duke Math. J. 10 (1943), 309-333], we say that a group $G$ is strongly resolvable if for every nondiscrete Hausdorff group topology $\mathscr{T}$ on $G$ there is $D \subseteq G$ such that both $D$ and $G \backslash D$ are $\mathscr{T}$-dense in $G$.
\end{abstract}

Theorem. Let $G$ be an Abelian group.

(a) If $G$ contains no subgroup isomorphic to the group $\bigoplus_{\omega}\{0,1\}$, then $G$ is strongly resolvable.

(b) Assume MA. If $G$ contains a copy of $\bigoplus_{\omega}\{0,1\}$, then $G$ is not strongly resolvable.

Our proof of (b) depends heavily on work of Malykhin.

\section{NOTATION AND TERMINOLOGY}

The cardinality of a set $X$ is written $|X|$.

In dealing with Abelian groups we use additive notation and we denote the neutral element by the symbol 0 (with a subscript, when this seems necessary).

The symbols $\mathbb{Z}$ and $\mathbb{Q}$ denote as usual the sets of integers and rational numbers, respectively; $\mathbb{P}=\{p \in \mathbb{Z}: p>1, p$ is prime $\}$; and $\mathbb{Z}\left(p^{\infty}\right)$ for $p \in \mathbb{P}$ is the Prüfer group

$$
\mathbb{Z}\left(p^{\infty}\right)=\bigcup_{n<\omega}\left\{x \in \mathbb{Q} / \mathbb{Z}: p^{n} x=0\right\} .
$$

For an Abelian group $G$ the torsion-free rank of $G$ and (for $p \in \mathbb{P}$ ) the $p$ rank of $G$ are denoted $r_{0}(G)$ and $r_{p}(G)$, respectively. We are concerned in particular with the 2-rank $r_{2}(G)$. Our notation is defined in order to ensure that for an Abelian group $G$ the following three conditions are equivalent:

(a) $G$ contains isomorphically a copy of the group $\bigoplus_{\omega}\{0,1\}$.

(b) $r_{2}(G) \geq \omega$.

(c) Infinitely many elements $x$ of $G$ satisfy $2 x=0$.

Received by the editors June 24, 1992.

1991 Mathematics Subject Classification. Primary 20K45, 22A05, 54H11, 54G05; Secondary $03 E 35$.

Key words and phrases. Dense subset, resolvable topological space, strongly resolvable group, Boolean group, extremally disconnected space, extremally disconnected topological group, maximal topology, Abelian group, divisible hull.

The second author is pleased to thank the Department of Mathematics of Wesleyan University for generous hospitality and support during the spring semester of 1992. 
We use these three properties interchangeably.

A Boolean group is a (necessarily Abelian) group in which every element $x$ satisfies $2 x=0$.

Throughout this paper, expressions like "topological group" and "group topology" (on a group $G$ ) refer always to Hausdorff topological groups. As is well known (see, e.g., $[11,8.4]$ ), this guarantees that our topological groups are Tychonoff spaces, i.e., completely regular, Hausdorff spaces.

Following Hewitt [10], we say that a topological space $X=\langle X, \mathscr{T}\rangle$ is resolvable if there is $D \subseteq X$ such that both $D$ and $X \backslash D$ are $\mathscr{T}$-dense in $X$. Hewitt [10] showed that irresolvable Tychonoff spaces (without isolated points) exist in profusion; indeed, according to [10], every Tychonoff space $\langle X, \mathscr{U}\rangle$ without isolated points admits an irresolvable Tychonoff "expansion" $\mathscr{T} \supseteq \mathscr{U}$ such that $\langle X, \mathscr{T}\rangle$ has no isolated points.

We say that a group $G$ is strongly resolvable if for every nondiscrete group topology $\mathscr{T}$ on $G$ the space $\langle G, \mathscr{T}\rangle$ is resolvable. We recall in this connection that it has been shown by Hesse [9], Shelah [16] (assuming $\mathrm{CH}$ ), and Ol'shanskii [14] using algebraic constructs of Adian [1], that there are infinite (non-Abelian) groups $G$ with no group topology except the discrete topology; evidently all such groups, and all finite groups, are strongly resolvable.

\section{INTRODUCTION}

The question whether every nondiscrete topological group admits a proper dense subgroup has been examined in various contexts in the literature. For example, every infinite connected Abelian group, every infinite pseudocompact group, and every uncountable totally bounded Abelian torsion-free group $G$ of weight $|G|$ admits a proper dense subgroup [7]. And every pseudocompact Abelian group $G$ whose weight $w G$ is uncountable admits a proper dense pseudocompact subgroup, provided that either $G$ is zero dimensional [6], or $|G|>\mathfrak{c}[4]$, or $w G \leq \mathfrak{c}$ [4]. In the negative direction, there are nondiscrete $\omega$-bounded (hence, countably compact) groups with no proper dense countably compact subgroup [6,3.3]; there are nondiscrete, locally compact Abelian groups (of arbitrary infinite local weight) with no proper dense subgroup [15]; and for suitably restricted uncountable cardinals $\alpha$ there are totally bounded Abelian torsion groups $G$ of weight $\alpha=|G|$ such that $G$ has no proper dense subgroup [7].

Briefly, then, the problem of classifying those topological groups which have a proper dense subgroup is far from settled.

The foregoing remarks suggest two less ambitious problems:

(I) Classify the resolvable topological groups.

(II) Classify the strongly resolvable groups.

Though our paper contributes a bit to the first of these problems, it remains substantially unsolved. Our principal result, which is a definitive solution to the second problem for Abelian groups provided that MA is assumed, reads as follows.

Theorem. Let $G$ be an Abelian group.

(a) If $G$ contains no subgroup isomorphic to the group $\bigoplus_{\omega}\{0,1\}$, then $G$ is strongly resolvable. 
(b) Assume MA. If $G$ contains a copy of $\bigoplus_{\omega}\{0,1\}$, then $G$ is not strongly resolvable.

(Evidently, then, under MA an Abelian group $G$ is strongly resolvable if and only if $r_{2}(G)<\omega$.)

We do not know whether this theorem remains true if assumption MA is omitted. We discuss this question and several related questions in $\S 6$.

We proceed according to the following strategy. In $\S 3$ we assemble several very general facts about resolvable spaces and resolvable topological groups. $\S 4$ shows specifically that the groups $\mathbb{Z}\left(p^{\infty}\right)$ and $\mathbb{Q}$ are strongly resolvable. In $\S 5$ we reduce the problem of classifying strongly resolvable Abelian groups to the divisible case and we apply standard structure theory and our results on $\mathbb{Z}\left(p^{\infty}\right)$ and $\mathbb{Q}$ to solve that problem under MA. Questions and discussion are postponed to $\S 6$.

\section{General Results}

Much of 3.1-3.8 inclusive is immediate from the original work of Hewitt [10] (although [10] did not explicitly consider resolvability in the context of topological groups). 3.1-3.4 are taken from [4]; we include proofs here for the reader's convenience, in order that this paper be self-contained.

3.1. Lemma. Let $X=\langle X, \mathscr{T}\rangle$ be a space. If $X$ has the form $X=\bigcup_{i \in I} X_{i}$ with $\left\{X_{i}: i \in I\right\}$ a pairwise disjoint family such that each $X_{i}$ is resolvable (in the topology inherited from $\langle X, \mathscr{T}\rangle)$, then $X$ itself is resolvable.

Proof. For $i \in I$ there is $D_{i} \subseteq X_{i}$ such that both $D_{i}$ and $X_{i} \backslash D_{i}$ are dense in $X_{i}$. Then $\bigcup_{i \in I} D_{i}$ and its complement are both $\mathscr{T}$-dense in $X$.

Remark. Of course in 3.1 the assumption $X=\bigcup_{i \in I} X_{i}$ can be weakened to the condition that $\bigcup_{i \in I} X_{i}$ is dense in $\langle X, \mathscr{T}\rangle$.

3.2. Lemma. Let $G=\langle G, \mathscr{T}\rangle$ be a topological group with a subgroup $H$ such that $H$ is resolvable (in the topology inherited from $\langle G, \mathscr{T}\rangle$ ). Then $G$ is resolvable.

Proof. Each coset of $H$ in $G$ is resolvable, so 3.1 applies.

3.3. Lemma. A topological group $G=\langle G, \mathscr{T}\rangle$ with a proper dense subgroup is resolvable.

Proof. Evidently $H$ and $G \backslash H$ are $\mathscr{T}$-dense in $G$.

3.4. Lemma. A topological group $G=\langle G, \mathscr{T}\rangle$ with a nonclosed subgroup $H$ is resolvable.

Proof. The group $\bar{H}$ is resolvable by 3.3, so 3.1 applies.

3.5. Lemma. Let $G$ be a topological group with a closed, nowhere dense subgroup $H$ such that $G / H$ is resolvable (in the usual quotient topology). Then $G$ is resolvable.

Proof. Let $\phi: G \rightarrow G / H$ be the natural map, and let $D$ and $(G / H) \backslash D$ be complementary dense subsets of $G / H$. It is easy to check, using the fact that $\phi$ is an open map (cf. $[11,5.17])$, that $\phi^{-1}(D)$ and $G \backslash \phi^{-1}(D)$ are $\mathscr{T}$-dense in $G$. 
We remark that in 3.5 it is not required that $H$ or $\bar{H}$ be normal subgroups of $G$.

When $H$ is a subgroup of an Abelian group $G$ and $H$ has a group topology $\mathscr{U}$, one may define a group topology $\mathscr{T}$ on $G$ by declaring that $\langle H, \mathscr{U}\rangle$ is an open topological subgroup of $\langle G, \mathscr{T}\rangle$. The following statement, whose proof we leave to the reader, gives the specifics. See also Hewitt and Ross [11, 4.5] for a more general result.

3.6. Lemma. Let $H$ be a subgroup of an Abelian group $G$, and let $\mathscr{U}$ be a group topology for $H$. Let $\mathscr{T}$ be the family of subsets of $G$ defined as follows: $A$ subset $A$ of $G$ satisfies $A \in \mathscr{T}$ if and only if every $x \in A$ satisfies $x^{-1} A \cap H \in \mathscr{U}$. Then $\langle G, \mathscr{T}\rangle$ is a topological group.

3.7. Corollary. Let $H$ be a group which is not strongly resolvable, and let $G$ be an Abelian group containing $H$ as a normal subgroup. Then $G$ is not strongly resolvable.

Proof. Let $\mathscr{U}$ be a (nondiscrete) irresolvable group topology on $H$, and define $\mathscr{T}$ as in the statement of 3.6. Then $\mathscr{T}$ is a nondiscrete, irresolvable group topology on $G$, as required.

3.8. Theorem. Let $G$ be a group with a normal subgroup $H$ such that both $H$ and $G / H$ are strongly resolvable. Then $G$ is strongly resolvable.

Proof. Let $\mathscr{T}$ be a nondiscrete group topology for $G$. We consider three cases.

Case 1. $H$ is not $\mathscr{T}$-closed in $G$. Then the desired conclusion (that $G$ admits complementary $\mathscr{T}$-dense subsets) follows from 3.4 .

Case 2. The topology induced by $\mathscr{T}$ on $H$ is not discrete. The desired conclusion now follows from 3.2.

Case 3. Cases 1 and 2 fail. Since $H$ is closed, the quotient topology $\mathscr{T}_{q}$ on the group $G / H$ makes $G / H$ a topological group (i.e., the Hausdorff separation axiom is satisfied). The group $H$ has no $\mathscr{T}$-interior in $G$, since otherwise $H$ is open and $\langle G, \mathscr{T}\rangle$ itself is discrete, so $\left\langle G / H, \mathscr{T}_{q}\right\rangle$ is not discrete. The desired conclusion then follows from 3.5.

3.9. Corollary. Let $G_{0}$ and $G_{1}$ be strongly resolvable groups, and let $G=$ $G_{0} \times G_{1}$. Then $G$ is strongly resolvable.

Proof. It is enough to take $H=G_{0}, G / H=G_{1}$ in 3.8 .

3.10. Remark. It is good to notice that a product $G=G_{0} \times G_{1}$ may carry a nondiscrete group topology $\mathscr{T}$ which nevertheless induces the discrete topology on both $G_{0}$ and $G_{1}$. For example, let $G_{0}=G_{1}=H$ be an infinite Abelian group, let $\mathscr{U}$ be a nondiscrete group topology on $H$ (these exist in profusion: see $[5, \S \S 3.5,3.10 \mathrm{I}]$ for a survey of the literature), and note that the natural "diagonal" copy $\Delta=\langle\Delta, \mathscr{U}\rangle$ of $H$ inside $G=H \times H=G_{0} \times G_{1}$ is a normal subgroup of $G$. The group topology $\mathscr{T}$ defined on $G$ by the topological group $\langle\Delta, \mathscr{U}\rangle$ (as in 3.6) is not discrete, but the topology induced by $\mathscr{T}$ on $G_{0}$ and $G_{1}$ is the discrete topology.

We view our next result as a generalization of 3.9. In its statement, the hypothesis concerning the group $\bigoplus_{\omega}\{0,1\}$ may strike the reader as bizarre, capricious, or irrelevant. The role of this assumption will be clarified in 5.5, but for the moment two observations may be helpful: 
(1) Our proof does not apply and cannot be restructured to apply to the case $G \supseteq \bigoplus_{\omega}\{0,1\}$.

(2) According to work of Malykhin [13], the conclusion of 3.11 fails for $G=\bigoplus_{\omega}\{0,1\}$ in certain models of ZFC.

3.11. Theorem. Let $\kappa$ be a cardinal number, and let $\left\{G_{\xi}: \xi<\kappa\right\}$ be a set of (not necessarily distinct) strongly resolvable Abelian groups. Let $G=\bigoplus_{\xi<\kappa} G_{\xi}$, and suppose that $r_{2}(G)<\omega$; that is, $G$ contains no copy of the group $\bigoplus_{\omega}\{0,1\}$. Then $G$ is strongly resolvable.

Proof. Arguing by induction over the class of cardinals, we assume that $\kappa$ is the least cardinal for which the statement fails; from 3.9 we have $\kappa \geq \omega$.

We assume that each $\xi<\kappa$ satisfies $\left|G_{\xi}\right|>1$.

Let $\mathscr{T}$ be a nondiscrete group topology for $G$. To show that $\langle G, \mathscr{T}\rangle$ is resolvable, we consider two cases.

Case 1. There exist a neighborhood $V$ of 0 and $\eta<\kappa$ such that $V \subseteq$ $\bigoplus_{\xi<\eta} G_{\xi}$. Then, because $\langle G, \mathscr{T}\rangle$ is nondiscrete, the topology induced by $\mathscr{\mathscr { T }}$ on $\bigoplus_{\xi<\eta} G_{\eta}$ is nondiscrete. Since $|\eta|<\kappa$, the group $\bigoplus_{\xi<\eta} G_{\xi}$ is strongly resolvable by the inductive assumption, so the group $\langle G, \mathscr{T}\rangle$ is resolvable by 3.2.

Case 2. Case 1 fails. For $\xi<\kappa$ set

$$
F_{\xi}=\left\{x_{\xi} \in G_{\xi}: 2 x_{\xi}=0_{\xi}\right\}
$$

and use Zorn's lemma to find a subset $D_{\xi}$ of $G_{\xi}$ maximal with respect to the property $D_{\xi} \cap-D_{\xi}=\varnothing$. Then $D_{\xi} \cap F_{\xi}=\varnothing$, and since every element $x \in G_{\xi} \backslash F_{\xi}$ satisfies either $x \in D_{\xi}$ or $-x \in D_{\xi}$, we have $D_{\xi} \cup\left(-D_{\xi}\right) \cup F_{\xi}=G_{\xi}$.

Now for $0 \neq x=\left\langle x_{\xi}\right\rangle_{\xi<x} \in G$ set

$$
s(x)=\left\{\xi: x_{\xi} \neq 0_{\xi}\right\} \quad \text { and } \quad m(x)=\max s(x)
$$

and define

$$
D=\left\{x \in G: x \neq 0, x_{m(x)} \in D_{m(x)}\right\} .
$$

We will show in this case that both $D$ and $G \backslash D$ are $\mathscr{T}$-dense in $G$.

Let $0 \neq x \in U \in \mathscr{T}$, and let $V$ be a neighborhood of $0 \in G$ such that $V=-V$ and $x+V \subseteq U$.

We note that the set $\left\{\xi<\kappa:\left|F_{\xi}\right|>1\right\}$ is bounded above. (For otherwise from $\kappa \geq \omega$ it follows that $r_{2}\left(G_{\xi}\right) \geq 1$ for infinitely many $\xi$, and we have the contradiction $r_{2}(G) \geq \omega$.)

It then follows that there is $y \in V$ such that $m(y)>m(x)$ and $y_{m(y)} \notin$ $F_{m(y)}$. Of the two points $y_{m(y)}$ and $(-y)_{m(y)}$, exactly one lies in $D_{m(y)}$ (and the other in $\left.G_{m(y)} \backslash D_{m(y)}\right)$. From $(x+y)_{m(x+y)}=y_{m(y)}$ and $(x-y)_{m(x-y)}=(-y)_{m(y)}$ it follows that exactly one of the two points $x+y$ and $x-y$ lies in $D$ (the other in $G \backslash D)$. The observations $x+y \in x+V \subseteq U$, and $x-y \in x-V=x+V \subseteq U$ complete the proof.

\section{SPECIFIC RESULTS}

As indicated earlier, we show here that the group $\mathbb{Q}$ of rationals and the Prüfer groups $\mathbb{Z}\left(p^{\infty}\right)$ (with $p \in \mathbb{P}$ ) are strongly resolvable.

We remind the reader of a consequence of our additive notation: When $G$ is an Abelian group, $A \subseteq G$, and $n>0$, we have $n A=\left\{x_{1}+x_{2}+\cdots+x_{n}: x_{i} \in A\right\}$. It follows in particular that if $0 \in A$ then $A \subseteq k A \subseteq n A$ for $1 \leq k \leq n$.

Our first result is taken from [4]. 
4.1. Theorem. The group $\mathbb{Z}$ is strongly resolvable.

Proof. Let $D=\{x \in \mathbb{Z}: x>0\}$. We show that for every nondiscrete group topology $\mathscr{T}$ on $\mathbb{Z}$ the sets $D$ and $\mathbb{Z} \backslash D$ are $\mathscr{T}$-dense.

Let $\varnothing \neq U \in \mathscr{T}$, and suppose that $U \subseteq D$. Set $m=\min U$. Since the maps $x \rightarrow-x$ and $x \rightarrow x+2 m$ are $\mathscr{T}$-homeomorphisms, the set

$$
U \cap(-U+2 m)=\{m\}
$$

is $\mathscr{T}$-open in $\mathbb{Z}$. Thus $\langle\mathbb{Z}, \mathscr{T}\rangle$ is discrete, a contradiction.

A similar argument shows that the relation $U \subseteq \mathbb{Z} \backslash D$ is also impossible.

For $G$ an Abelian group and $x$ a torsion element of $G$, we denote the order of $x$ by the symbol $o(x)$. We realize the Prüfer group $\mathbb{Z}\left(p^{\infty}\right)$ as a subgroup of $\{x: 0 \leq x<1\}$ with addition modulo 1 , and for $0 \neq x \in \mathbb{Z}\left(p^{\infty}\right)$ with $o(x)=p^{k}$ we write

$$
x=\sum_{n=1}^{k} \frac{a_{n}}{p^{n}}
$$

with $0 \leq a_{i}<p$ for $1 \leq i \leq k, a_{k} \neq 0$.

For $x$ as in $(*)$ the support of $x$ is the set $s(x)=\left\{n: a_{n} \neq 0\right\}$; we write $m(x)=k=\max s(x)$, so $o(x)=p^{m(x)}$.

4.2. Lemma. For $p \in \mathbb{P}$ the group $\mathbb{Z}\left(p^{\infty}\right)$ is strongly resolvable.

Proof. Fix $p \in \mathbb{P}$, and let $D=\left\{x \in \mathbb{Z}\left(p^{\infty}\right): x \neq 0, m(x)\right.$ is even $\}$. We will show that $D$ and $\mathbb{Z}\left(p^{\infty}\right) \backslash D$ are dense in every nondiscrete group topology $\mathscr{T}$ on $\mathbb{Z}\left(p^{\infty}\right)$.

Let $\pi=\pi_{p}: \mathbb{Z}\left(p^{\infty}\right) \rightarrow \mathbb{Z}\left(p^{\infty}\right)$ be the homomorphism defined by $\pi(x)=p x$ $\left(x \in \mathbb{Z}\left(p^{\infty}\right)\right)$.

Let $x \in U \in \mathscr{T}$, and let $V$ be a neighborhood of 0 such that $x+p V \subseteq U$. We claim that there is $y \in V$ such that $m(y)>m(x)+2$. Indeed, otherwise $V$ is a subset of the finite set $\left\{z \in \mathbb{Z}\left(p^{\infty}\right): m(z) \leq m(x)+2\right\}$, so $\mathscr{T}$ is the discrete topology on $\mathbb{Z}\left(p^{\infty}\right)$; this contradiction establishes the claim. Choosing such $y$, we have $m(x+y)=m(y)$, while $m(x+p y)=m(p y)=m(y)-1$. Of the two integers $m(y)=m(y)-1$, exactly one is even and exactly one is odd. Since $x+y \in x+V \subseteq U$ and $x+p y \in x+p V \subseteq U$, it is clear that $U$ meets both $D$ and $\mathbb{Z}\left(p^{\infty}\right) \backslash \bar{D}$, as required.

4.3. Corollary. The group $\bigoplus_{p \in \mathbb{P}} \mathbb{Z}\left(p^{\infty}\right)$ is strongly resolvable.

Proof. Since each group $\mathbb{Z}\left(p^{\infty}\right)$ is strongly resolvable by Lemma 4.2 and $r_{2}\left(\bigoplus_{p \in \mathbb{P}} \mathbb{Z}\left(p^{\infty}\right)\right)=r_{2}\left(\mathbb{Z}\left(2^{\infty}\right)\right)=1<\omega$, this is immediate from 3.11 .

4.4. Theorem. The group $\mathbb{Q}$ is strongly resolvable.

Proof. A standard structure theorem (see, e.g., Fuchs [8, p. 43]) gives the isomorphism $\mathbb{Q} / \mathbb{Z} \approx \bigoplus_{p \in \mathbb{P}} \mathbb{Z}\left(p^{\infty}\right)$. Since $\mathbb{Z}$ and $\bigoplus_{p \in \mathbb{P}} \mathbb{Z}\left(p^{\infty}\right)$ are strongly resolvable by 4.1 and 4.3 , respectively, the required statement follows from 3.8.

The constituent groups being now under control, we proceed to put the pieces in place.

\section{Strongly Resolvable Abelian groups}

The following well-known structure theorem is basic to the study of divisible Abelian groups. 
See Fuchs $[8, \S \S 23,24]$ or Hewitt and Ross [11, A.14, A.16] for a proof and commentary.

5.1. Theorem. Every divisible Abelian group $K$ is isomorphic to the group

$$
\bigoplus_{\alpha_{0}} \mathbb{Q} \oplus \bigoplus_{p \in \mathbb{P}} \bigoplus_{\alpha_{p}} \mathbb{Z}\left(p^{\infty}\right)
$$

with $\alpha_{0}=r_{0}(K)$ and $\alpha_{p}=r_{p}(K)$ for $p \in \mathbb{P}$.

(Of course, when $\alpha_{0}=0$ or $\alpha_{p}=0$ the corresponding summand may be omitted in $(* *)$.)

Theorem 5.1 now gives the assertion we want for divisible Abelian groups.

5.2. Theorem. Every divisible Abelian group $K$ such that $r_{2}(K)<\omega$ is strongly resolvable.

Proof. This is immediate from 4.3, 4.4, 5.1, and (the case $\kappa=\alpha_{0}+\sum_{p \in \mathbb{P}} \alpha_{p}$ of) 3.11 .

Our principal positive "absolute" theorem now emerges in the following form.

5.3. Theorem. Every Abelian group $G$ such that $r_{2}(G)<\omega$ is strongly resolvable.

Proof. As is well known (see the above-mentioned passages in $[11,8]$ ), the divisible hull (that is, the minimal divisible extension) $\bar{G}$ of $G$ satisfies $G \subseteq \bar{G}$, and $r_{0}(\bar{G})=r_{0}(G)$ and $r_{p}(\bar{G})=r_{p}(G)$ for $p \in \mathbb{P}$. In particular, $r_{2}(\bar{G})=r_{2}(G)$, so $\bar{G}$ is strongly resolvable by 5.2 . That $G$ is strongly resolvable then follows from 3.7.

The axioms $\mathrm{CH}$ and MA are surely known to the reader. Slightly less familiar is the combinatorial principle $P(\mathfrak{c})$, a consequence of MA which states that if $\left\{A_{\xi}: \xi<\alpha\right\}$ is a family of subsets of $\omega$ such that every finite subfamily has infinite intersection and if $\alpha<\mathfrak{c}$, then there is infinite $A \subseteq \omega$ such that $\left|A \backslash A_{\xi}\right|<\omega$ for all $\xi<\alpha$.

A topological space $X=\langle X, \mathscr{T}\rangle$ is said to be extremally disconnected if the closure of every $\mathscr{T}$-open subset of $X$ is again $\mathscr{T}$-open. (An equivalent condition is that disjoint $\mathscr{T}$-open subsets of $X$ have disjoint closures.) It is an old question of Arkhangel'skii [2], still unsettled in ZFC, whether there exists a nondiscrete extremally disconnected topological group. The problem has been successfully addressed in augmented axiom systems: Using $\mathrm{CH}, \mathrm{MA}$, and $P(\mathrm{c})$ respectively, Sirota [17], Louveau [12], and Malykhin [13] have proved that the group $\bigoplus_{\omega}\{0,1\}$ admits a nondiscrete extremally disconnected group topology. Indeed, Malykhin [13] proves more: the group topology $\mathscr{T}$ on $\bigoplus_{\omega}\{0,1\}$ may be chosen maximal among all nondiscrete Hausdorff topologies in $\bigoplus_{\omega}\{0,1\}$. (The fact that a maximal nondiscrete Hausdorff space is extremally disconnected is easily proved: If $\langle X, \mathscr{T}\rangle$ is maximal but $\bar{U} \notin \mathscr{T}$ with $U \in \mathscr{T}$, then the topology $\mathscr{U}$ on $X$ generated by $\mathscr{T} \cup\{\bar{U}\}$ properly contains $\mathscr{T}$ and every non- $\mathscr{T}$-isolated point of $X$ remains non- $\mathscr{U}$-isolated.)

The relevance of Malykhin's theorem to our concerns is given by the following result. 
5.4. Lemma (Hewitt [10]). Let $\langle X, \mathscr{T}\rangle$ be a maximal nondiscrete Hausdorff space. Then $\langle X, \mathscr{T}\rangle$ is irresolvable.

Proof. Suppose that $D$ and $X \backslash D$ are $\mathscr{T}$-dense in $X$, and let $\mathscr{U}$ be the topology on $X$ generated by $\mathscr{T} \cup\{D\}$; that is, $\mathscr{U}=\mathscr{T} \cup\{U \cap D: U \in \mathscr{T}\}$. The inclusion $\mathscr{U} \supseteq \mathscr{T}$ is proper, and it is easy to check that every non- $\mathscr{T}$-isolated point of $X$ is non- $\mathscr{U}$-isolated.

In view of 5.4, Malykhin's theorem has this consequence.

5.5. Corollary (Malykhin [13]). Assume $P(\mathfrak{c})$. The group $\bigoplus_{\omega}\{0,1\}$ admits a nondiscrete irresolvable group topology.

Combining 5.5 with 5.3 gives the result quoted in our abstract.

5.6. Theorem. Assume $P(\mathfrak{c})$ (or assume $\mathrm{MA}$ ), and let $G$ be an Abelian group. Then $G$ is strongly resolvable if and only if $r_{2}(G)<\omega$.

Proof. The statement "if" is given by 5.3 (without the additional assumption $P(\mathfrak{c}))$, while "only if" follows from 5.5 and 3.7 .

\section{DisCUSSION AND QUESTIONS}

We have shown that in the system $\mathrm{ZFC}+\mathrm{MA}$ an Abelian group $G$ is strongly resolvable if and only if $r_{2}(G)<\omega$. This result raises some questions, as follows.

6.1. Question. In the system ZFC (with no additional axioms), can the group $\bigoplus_{\omega}\{0,1\}$ be given a nondiscrete extremally disconnected group topology?

6.2. Question. If a (possibly non-Abelian) group $G$ contains a copy of $\bigoplus_{\omega}\{0,1\}$, can $G$ be given a nondiscrete extremally disconnected group topology? A nondiscrete irresolvable group topology? What if MA is assumed?

In connection with 6.1 it should be mentioned that Malykhin [13] has shown in ZFC that every group with a nondiscrete extremally disconnected group topology contains for some cardinal $\kappa \geq \omega$ an open-and-closed (hence, nondiscrete and extremally disconnected) subgroup isomorphic to $\bigoplus_{\kappa}\{0,1\}$. Accordingly, we ask additional questions.

6.3. Question. Does every nondiscrete irresolvable topological group $G$ contain an open-and-closed Boolean subgroup? What if $G$ is assumed Abelian?

6.4. Question. Is every irresolvable topological group $G$ extremally disconnected? What if $G$ is Abelian?

6.5. Question. Suppose that a topological group $G$ contains a (not necessarily open) subset $D$ such that $0 \in \overline{D \backslash\{0\}} \cap \overline{(G \backslash D) \backslash\{0\}}$. Must $G$ be resolvable?

6.6. Remarks. (a) In work with Gladdines [4] we have shown that every Abelian totally bounded group is resolvable. It is reassuring to note the availability of an elementary proof that this result is not in conflict with the constructions of Sirota, Louveau, and Malykhin. Briefly, an infinite extremally disconnected group is not totally bounded. To see this, suppose that $G$ is a counterexample, and let $K$ be the (compact) Weil completion of $G$. Then $K$ is extremally disconnected since otherwise some point of $K$ (hence, every point of $K$ ) is in 
the closure of disjoint open subsets of $K$, so every point of $G$ is in the closure of disjoint open subsets of $G$. Thus $K$ is a compact, extremally disconnected homogeneous space and, hence, is finite, so $G$ is finite.

(b) It cannot be proved in ZFC that every extremally disconnected group is irresolvable. Indeed the extremally disconnected group constructed by Louveau [12] using MA is defined on the underlying set of finite subsets of $\omega$, with "multiplication" given by the symmetric difference operation $A \cdot B=A \Delta B=$ $(A \backslash B) \cup(B \backslash A)$. As in [17], Louveau's group $G$ is topologized so that every neighborhood of each $A \in G$ contains for each $n>|A|$ elements $B$ of $G$ such that $|B|=n$. It is then clear that in the Louveau group the set $\{X \subseteq \omega:|X|<\omega$ and $|X|$ is even $\}$ and its complement are both dense. Thus Louveau's group is resolvable (and hence is not maximal among nondiscrete Hausdorff spaces).

(c) Let us say that a subset $D$ of a group $G$ is absolutely dense if $D$ is dense in every nondiscrete group topology on $G$, and $G$ is absolutely resolvable if $G$ admits complementary absolutely dense subsets. Of course, an absolutely resolvable group is strongly resolvable. In 4.1 and 4.2 we showed that the groups $\mathbb{Z}$ and $\mathbb{Z}\left(p^{\infty}\right)$ (for $p \in \mathbb{P}$ ) are absolutely resolvable. This suggests the following problem.

\subsection{Question. Characterize algebraically the absolutely resolvable groups.}

6.8. Remark (added November, 1992). One of us has noted recently with Feng [3] that Lemma 3.1 can be improved as follows: Each space $X$ of the form $X=$ $\bigcup_{i \in I} X_{i}$ with each $X_{i}$ resolvable is itself resolvable. (The proof is elementary. A maximal family $\mathscr{A}$ of pairwise disjoint resolvable subspaces of $X$ must have dense union, since otherwise some $i$ satisfies $\left.X_{i} \backslash \overline{\cup \mathscr{A}}^{X}\right) \neq \varnothing$, and then $X_{i} \backslash \overline{\cup \mathscr{A}}^{X}$ may be adjoined to $\mathscr{A}$. Then $\bigcup \mathscr{A}$ is resolvable by 3.1 , so $X$ is resolvable.) As is noted in [3], it is immediate that a topological group with a nonempty resolvable subspace is itself resolvable. This observation seems to have no direct impact on this paper, but it may suggest an avenue of approach to some of the questions cited above.

\section{ACKNOWLEDGMENT}

We are grateful to Sophia U. Trigos for pointing out several errors and ambiguities in an early version of this manuscript.

\section{REFERENCES}

1. S. I. Adian, Classifications of periodic words and their application in group theory, Burnside Groups (Proc. Bielefeld, Germany 1977 Workshop) (J. L. Mennicke, ed.), Lecture Notes in Math., vol. 806, Springer-Verlag, Berlin, 1980, pp. 1-40.

2. A. V. Arkhangel'skii, Every extremally disconnected bicompactum of weight $\mathrm{c}$ is inhomogeneous, Dokl. Akad. Nauk SSSR 175 (1967), 751-754; English transl., Soviet Math. Dokl. 8 (1967), 897-900.

3. W. W. Comfort and Li Feng, The union of resolvable spaces is resolvable, Math. Japon 38 (1993), 413-414.

4. W. W. Comfort, Helma Gladdines, and Jan van Mill, Proper pseudocompact subgroups of pseudocompact Abelian groups, Proc. Eighth Summer Topology Conference (Queen's College, New York, June, 1992); Ann. New York Acad. Sci. (to appear). 
5. W. W. Comfort, K. H. Hofmann, and D. Remus, Topological groups and semigroups, Recent Progress in General Topology (M. Hušek and J. van Mill, eds.), North-Holland, Amsterdam, 1992, pp. 57-144.

6. W. W. Comfort and Lewis C. Robertson, Extremal phenomena in certain classes of totally bounded groups, Dissertationes Math. (Rozprawy Mat.) 272 (1988).

7. W. W. Comfort and Jan van Mill, Some topological groups with, and some without, proper dense subgroups, Topology Appl. 41 (1991), 3-15.

8. Lásló Fuchs, Infinite Abelian groups, Academic Press, New York, San Francisco, and London, 1970.

9. G. Hesse, Zur Topologisierbarkeit von Gruppen, Ph.D. thesis, Universität Hannover, Hannover, Germany, 1979.

10. Edwin Hewitt, A problem of set-theoretic topology, Duke Math. J. 10 (1943), 309-333.

11. Edwin Hewitt and Kenneth A. Ross, Abstract harmonic analysis, vol. I, Grundlehren Math. Wiss., vol. 15, Springer-Verlag, Berlin, Göttingen, and Heidelberg, 1963.

12. Alain Louveau, Sur un article de S. Sirota, Bull. Sci. Math. (2) 96 (1972), 3-7.

13. V. I. Malykhin, Extremally disconnected and similar groups, Dokl. Akad. Nauk SSSR 230 (1975), 27-30; English transl., 16 (1975), 21-25.

14. A. Yu. Ol'shanskii, A remark on a countable non-topologizable group, Vestnik Moskov. Univ. Ser. I Mat. Mekh. 3 (1980), 103. (Russian).

15. M. Rajagopalan and H. Subrahmanian, Dense subgroups of locally compact groups, Colloq. Math. 35 (1976), 289-292.

16. Saharon Shelah, On a problem of Kurosh, Jonsson groups and applications, Word Problems II (S. I. Adian, W. W. Boone, and G. Higman, eds.), North-Holland, Amsterdam, 1980, pp. 373-394.

17. S. M. Sirota, The product of topological groups and extremal disconnectedness, Mat. Sb. 79 (121) (1969), 179-192; English transl., Math. USSR-Sb. 8 (1969), 169-180.

Department of Mathematics, Wesleyan University, Middletown, Connecticut 06459 E-mail address: :comfort@eagle. vesleyan.edu

Faculteit Wiskunde en Informatica, VRue Universiteit, De BoelelaAn 1081A, 1081 HV AMSTERDAM, THE NETHERLANDS

E-mail address: vanmillecs.vu.nl 